\title{
Functional Building Blocks for an Integrated Aeronautical IP-Network
}

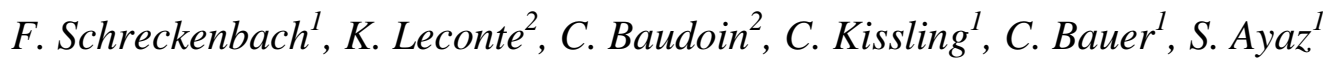 \\ ${ }^{1}$ German Aerospace Center (DLR), 82234 Wessling, Germany \\ ${ }^{2}$ Thales Alenia Space, 31037 Toulouse Cedex 1, France
}

\begin{abstract}
Within the next 10-15 years, Air-Traffic Management (ATM) will be primarily based on data communications and voice communication will be mostly used as fallback solution. Furthermore, it is foreseen that IP-based networking solutions for A/G communication will be deployed for cost savings, high reliability and an optimal alignment with the evolution of communication and security technologies. Such an efficient and sustainable data network for aeronautical communications is required to enable the implementation of a range of operational improvements needed to support the expected growth in air transportation. An IP-based global aeronautical communication network is currently being developed within the NEWSKY project, co-funded by the European Commission and in close collaboration with Eurocontrol and ICAO ACP WG-I (Specification of ATN/IPS). NEWSKY pursues the vision of "Networking the Sky" by integrating different data link technologies (long range A/G links, airport links, satellite links) and different services (ATS, AOC/AAC, APC) in a single, seamless network. Key functionalities have been identified, namely resource management, mobility, security and end-to-end data transport. In this paper, the raw functional architecture and the ongoing activities towards the specification of the functional modules are investigated.
\end{abstract}

\section{Introduction}

\section{Trends in Aeronautical Communications}

In view of the expected saturation of ATM communications by 2020-2025 due to air traffic increase, the envisaged paradigm shift in ATM as developed in SESAR, and the existing high market demand for passenger communications, the major trends in aeronautical communications are:
- Network-Centric ATM Operations will have a predominant role for the global provision of distributed services for Collaborative Decision Making (CDM) using the System Wide Information Management (SWIM) concept.

- Air Traffic Services (ATS) will be primarily based on highly safety-related data communication and voice communication will be mostly used as fallback solution.

- Airline Operational Communications (AOC) data traffic will strongly increase for efficient airline operations

- Air Passenger Communications (APC) systems are foreseen to be further developed to meet passengers' expectations of on board broadband communication services

Furthermore, different services, data links and networking solutions will be deployed:

The different services, ranging from ATS, AOC to APC shall coexist and partly or totally share the aeronautical network infrastructure as well as a number of data links, through adequate pre-emption policies to prioritize safety critical applications in front of non-safety critical applications. Different levels of integration are possible and subject to trade-off between costs, reliability and security.

According to AP17 - Future Communications Study (Eurocontrol/FAA) and SESAR, several data links are required to fulfill the ATM communication requirements: a satellite link (new standard developed within ESA IRIS program), an airport link (WiMAX), a high data rate air-ground link (L-DACS-1/2) and support of legacy data links (e.g. VDL2). Further data links for APC will be in operation. 
Currently, the Aeronautical Telecommunication Network (ATN) based on the ISO/OSI reference model as networking solution is being deployed (e.g. Link2000 program) to enable advanced services. However, due to the marginal deployment of the ISO/OSI protocols, operation and maintenance costs are considerable. When looking at the 2020 (and beyond) horizon, it is hence foreseen that the widely deployed IETF Internet Protocol Suite (IPS) networking solution will be deployed for A/G communication for cost savings, high reliability and an optimal alignment with the evolution of communication and security technologies. ICAO is currently specifying within WG-I such an ATN/IPS.

Summarizing, users of aeronautical communication services face a highly complex and fragmented communication architecture with different services, different data links and different networking solutions to be supported.

\section{NEWSKY Vision of "Networking the Sky"}

The aim of the NEWSKY project ${ }^{1}$ is to develop a concept and initial design of a global, seamless aeronautical communication network with focus on air-ground communications and IPv6 technologies. It defines and extends ATN/IPS capabilities, considering interoperability and the transition from ATN/OSI.

NEWSKY pursues the vision of "Networking the Sky" by integrating different data link technologies (long range A/G links, airport links, satellite links) and different services (ATS, AOC, APC) in a single, seamless network. Note that NEWSKY does not develop or adapt any new data links.

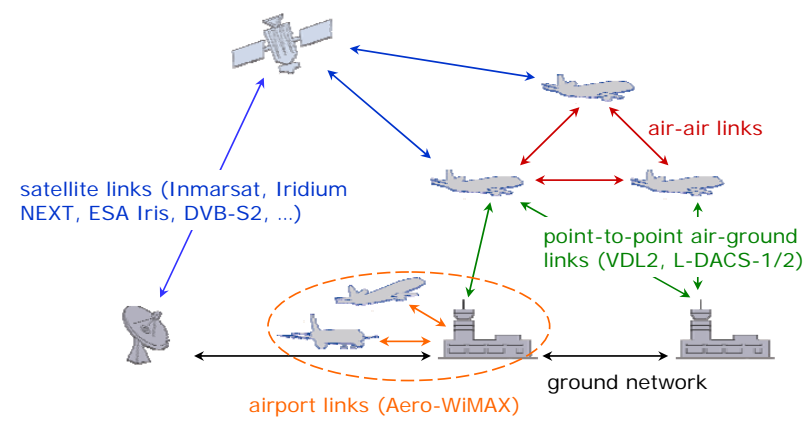

Figure 1. Integration of different data links into a global aeronautical communication network

\section{Relation to SESAR}

NEWSKY provides supporting CNS technology and is a system enabler for SESAR operational improvements. SESAR has not made a clear statement towards the revision of ATN/OSI or the deployment of a new ATN/IPS.

NEWSKY develops in close collaboration with Eurocontrol, ICAO ACP WG-I and relevant IETF WGs an initial design of an IPv6-based network for air/ground communications. Performance will be assessed through simulations and selected functionalities will be demonstrated in a laboratory test-bed to show the capabilities and the advantages the deployment of an ATN/IPS would encompass.

\section{NEWSKY Functional Building Blocks}

NEWSKY aims at developing a network supporting different services (ATS, AOC/AAC, APC) on different data links through the development or adaptation of network protocols and functionalities supporting this service integration.

Four major functionalities are identified to support such a network:

End-to-end data transport ensures the delivery of data end-to-end within the network.

Typically this function is supported by protocols such as TCP or some adaptations. As the NEWSKY network is possibly interconnected with different types of technologies (air/ground, air/air or satellite technologies) with very different performances in terms of delay, Bit Error Rate, etc ... this function need some adaptations depending on the technologies on which the data are sent.

Traffic management ensures traffic management (TM), Radio Resource Management (RRM) and support of Quality of Service (QoS).

This functionality is typically in charge of supporting the end-to-end quality of service for the different technologies, the traffic processing, scheduling and resource allocation at layer 1 and 2, and implementing (data or header) compression and congestion control (CC) mechanisms.

Mobility management supports the aircraft mobility throughout the network.

This functionality is split between the routing function that takes the decision and route the traffic on the correct path and technology, the handover 
function (HO) signaling the link quality and executing the handovers, and the network mobility function that handles mobility at network layer.

\section{Security.}

Security covers the main security functions (authentication, encryption, integrity and resistance to threats) and could be supported at different levels (physical, access, network, transport). However due to the contour of the NEWSKY project, security at physical and link access layer is not covered by the project.

The high level view of these network functionalities is presented in Figure 2. The representation is purely functional and not physical as the functions could impact or concern one or several aircraft, and the functions could be implemented in aircraft, on ground or both. Also some blocks (TM and RRM) must be implemented for each underlying technology used by the aircraft (multiple boxes on the figure).

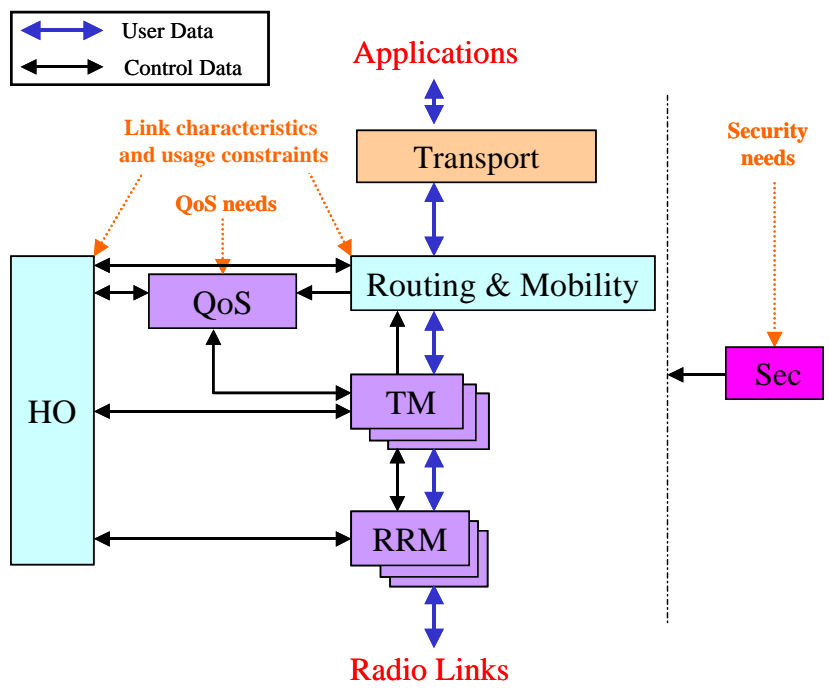

Figure 2. Overall network functionalities

The routing and $\mathrm{HO}$ decision is very central in such a network as it handles several data links and can perform a link switching due to poor quality foreseen on the used data links as well as some load balancing (re-assignment of traffic on another link in case the one used is overloaded). The handling of the overall set of data links with a single function allows to use very efficiently the different data links available in the aircraft even in case of overload or coverage shortage. This is a major advantage of the NEWSKY network.
The different functions are detailed in the next chapters.

\section{Traffic Management Functionality}

The traffic management functionality is split among 3 blocks named Quality of Service (QoS)”, Traffic Management (TM) and Radio Resource Management (RRM), see also Figure 1. Dependent on the QoS architecture which is chosen, the blocks fulfill different tasks or remain empty (see later).

The functional QoS block can be seen as a global functionality and shall ensure the provision and management of QoS on a global scale (not dedicated to a single aircraft or link). Besides the management of global QoS e.g. by means of bandwidth broking, a global Connection Admission Control (CAC) mechanism resides here.

The TM block manages the resources on per link or per technology base. The functionalities include management of CAC functionalities at layer 3 (L3) and QoS re-negotiation as explained in the sections about horizontal and vertical QoS mapping later on. Additionally the prevention of congestion situations (congestion control, CC) are addressed in this module. The CC information is then signaled to the Routing and Mobility block where this information is used to make a proper decision about the route and thus the link which shall be used. Finally the TM block does packet scheduling, queuing and traffic prioritization on L3 to ensure all data are sent in compliance with their requirements.

The RRM module works per link/ per technology as well as the TM block. This module takes care of lower layer functionalities such as allocation of resources and requesting capacity at access layer. Moreover it takes care of congestion control at access layer, i.e. per link. Besides the capacity allocation and congestion control, also the monitoring of the actual link status is performed here, a CAC mechanisms at link layer is implemented and efficient packet scheduling and framing at access layer is performed. To accomplish its tasks it is necessary to exchange information with the TM and HO modules. 
To explain the necessary QoS signaling and the applicable QoS architectures Figure 3 illustrates the NEWSKY network from a QoS perspective. It can be seen here that the network consists of different networks and domains which are interconnected. An end to end connection might traverse several network portions along the way. For provision of QoS it is necessary that all these portions can interoperate and have the same understanding of QoS.

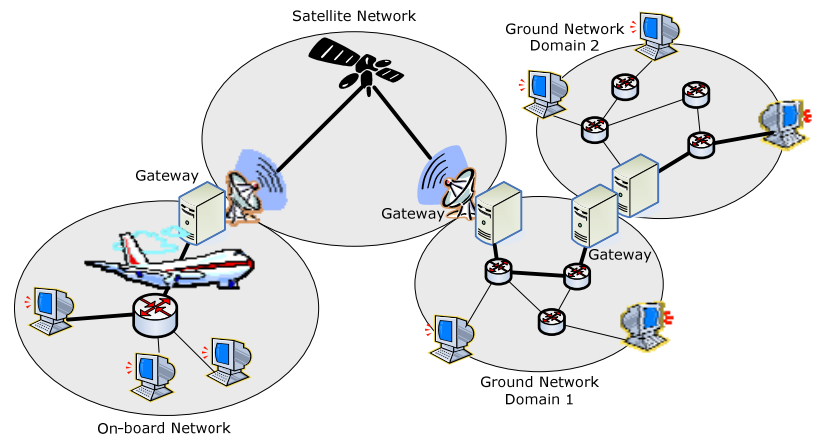

Figure 3: Illustration of the NEWSKY heterogeneity.

\section{Horizontal QoS mapping}

Horizontal QoS mapping addresses the QoS interoperability among the network portions. It is located in the traffic management modules from a functional perspective. According to reference ${ }^{2}$, the main problems of QoS interaction of different network portions in a heterogeneous network are, (1) to establish a proper communication interface among them, (2) to transfer the QoS needs for each end-to-end connection across the network portions in the path of the communication and (3) to map the QoS requests to the specific technology used in the network portion (vertical mapping, see next section). Horizontal QoS mapping has the task to ensure that the diverse network parts in the NEWSKY network can interoperate and communicate on a common base to manage QoS. Dependent on the used QoS architecture the signaling can include information about the network state and reservation of resources as for IntServ. An exhaustive presentation of all possible protocols is out of scope of this work for space reasons. An overview of possible signaling protocols is also provided in ${ }^{2}$.

\section{Vertical QoS mapping}

Different network portions will likely implement different physical and link layer technologies. For instance the protocols used in satellite segments can be different from the ones used for ad-hoc or VHF radio communication. The upper layers starting from L3 on the other hand can be assumed to be independent of the used link technologies in the focused NEWSKY ATN/IPS. Since for the provision of QoS the upper layers have to rely on the provision of QoS in the lower layers it is necessary to do a vertical mapping of Service Level Specifications down to the used technologies. The Broadband Satellite Multimedia (BSM) standard of ETSI $^{3}$ is dealing especially with this problem. Though the standard was developed in the context of satellite communication, its concepts can in principle be extended to any technology. Corresponding to ETSI-BSM the higher layers communicate with the lower layers via a so called Technology Independent Service Access Point (TISAP). The technology dependent (TD) link and physical layers then only have to implement their part of the interface of the TI-SAP. The communication is done via common "primitives". This architecture allows inserting or removing technologies without modification of the QoS mechanisms in the higher layers since the signaling is always done via the same primitives of the common TI-SAP interface.

Two of the major issues in vertical QoS mapping are the assignments of TI and TD priority queues or flows and the management of bandwidth and resources. For instance the higher layers may implement DiffServ with $m$ aggregates while the link technology just supports $n$ aggregates. In this case the TI-SAP has to map the $m$ higher layer queues in an appropriate way (i.e. complying with the latency and priority requirements) to the $n$ lower layer queues. The second issue which the control and resource management functionalities in the TISAP have to take care of is the mapping of TI bandwidth demand to TD bandwidth demand. The required bandwidth on the lower layers will in general be higher than the one seen at the upper layers due to encapsulation and technology specific overheads. 
QoS architectures and impact of several vs. one airborne router

For the overall management of QoS it must be decided which architectures shall be used. The most relevant and well known QoS architectures are IntServ (RFC $2205^{4}$, RFC $2210^{5}$ ), DiffServ (RFC $2475{ }^{6}$ ) and derivates or hybrid solutions ${ }^{2}$. The choice of the architecture also depends on whether one common airborne router is present which processes ATS/AOC as well as APC data or whether a separate router for ATS/AOC and another one for APC is considered.

In the case of one common router, it is necessary to ensure that the safety critical services (ATS/AOC) always get the required resources and never run into congestion and packet dropping. For this reason the implementation of a global resource management method like a DiffServ bandwidth broker or an IntServ resource reservation mechanism is necessary. These would then be located in the global QoS module. Additionally a CAC functionality is required to ensure that no APC connections are accepted which could impact the safety critical ATS/AOC communication by taking away bandwidth. The development of such a CAC mechanism is a complex task since not only the channel conditions are varying which make the estimation of the available capacity difficult, but also several heterogeneous links exist.

The situation simplifies if separated routers for ATS/AOC and APC are considered, e.g. for security and safety reasons. In this case no complex global resource management QoS architectures are necessary for ATS/AOC since the amount of available capacity has to be always sufficient. The task of resource management thus gets more a resource planning and dimensioning task and a simple static trunk DiffServ architecture can already provide the needed mechanisms to ensure message prioritization and transmission which is compliant with the requirements. Also no CAC is required in this case since rejection of connections is no option for ATS/AOC since they have to be always accepted. For APC on the other hand there are no stringent requirements so implementation of a general CAC is not meaningful here as well. For this reason the global QoS module does have no functionality for resource reservation and allocation since it is not necessary here. The global QoS thus implements no functionality for separated ATS/AOC and APC airborne routers.

\section{Mobility Functionality}

The routing module is closely interacting with the TM module regarding congestion control (CC) and, if applicable, call admission control (CAC). It has the following functions:

- Routing the traffic on the correct path depending on the HO decision (this part handles mobile ad hoc network (MANET) and inter-satellite link (ISL) routing tasks as well, if applicable.)

- Informing of the route retained (informing in particular the mobility function) so that network mobility can be implemented

- Managing the access network attachment

The mobility module is the second module responsible for performing mobility related signaling. NEWSKY has a heterogeneous network characteristic since it is composed of diverse terrestrial and satellite link technologies. Providing seamless mobility to the different user groups (i.e. cockpit users and passengers) is a challenging task in such network. According to the recent IETF progress on network layer mobility, two major fields of work were identified: scope of mobility and management of mobility as shown in Figure 4. The former notion introduces a hierarchical concept distinguishing between local and global mobility aspects; the latter addresses the functional entity responsible for mobility signaling. This can either be the mobile node $(\mathrm{MN})$ itself or the network performing signaling on behalf of the $\mathrm{MN}$.

The NEWSKY mobility solution is based on Mobile IPv6 (MIPv6 $\left.{ }^{1}\right)^{8}$ and its extensions. The extensions cover network-based local mobility (e.g. Proxy MIPv6 (PMIPv6) ${ }^{10}$ ), network mobility (NEMO) ${ }^{11}$ and multihoming support ${ }^{12}$.

\footnotetext{
${ }^{1}$ ICAO WG-I adopted MIPv6 as a global mobility management protocol for the ATN/IP in the 6th meeting ${ }^{9}$
} 


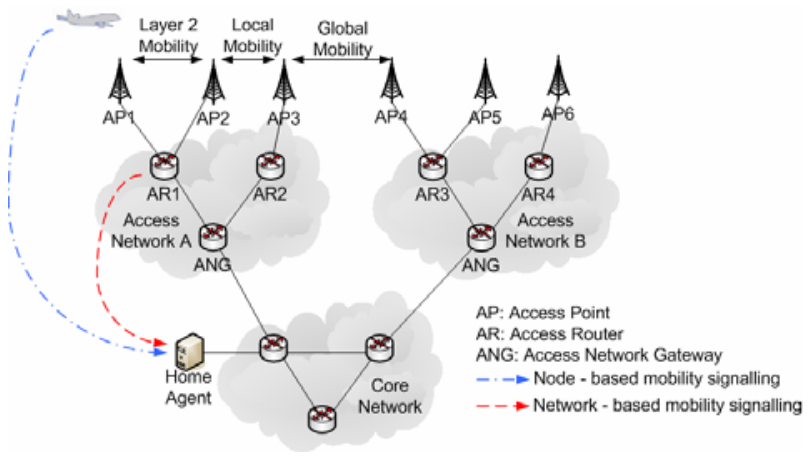

Figure 4. Levels of Mobility ${ }^{2}$

PMIPv6 is investigated since the protocol does not require an $\mathrm{MN}$ to perform mobility signaling as the network performs the required signaling on behalf of the MN. This is an attractive option since it significantly reduces the signaling overhead on the wireless link and provides better delay performance (since all the mobility signaling is performed in the wired links). However, the protocol provides only local mobility such that in case of an access network change, all ongoing sessions are broken. In order to maintain session continuity but also global reachability in such a scenario, MIPv6 functionality is needed for global mobility management.

NEMO is another promising extension to MIPv6 which provides mobility functionality to a group of mobile hosts. The NEMO Basic Support protocol ${ }^{11}$ is not providing route optimization which is very crucial in terms of delay considering the world wide movement of aircraft. Our investigations focused on different RO aspects ${ }^{13}$ for the aeronautical scenario (i.e. for cockpit users and passengers) considering the aeronautical requirements ${ }^{14}$.

As mentioned above, the NEWSKY network is utilizing diverse link technologies. In such an environment multihoming is another aspect one should consider. The old IETF WG MONAMI6 has worked on this issue and provided two documents which are: "Multiple Care-of Address (CoA) Registration" ${ }^{12}$ and "Flow Bindings in Mobile IPv6 and NEMO Basic Support" ${ }^{15}$. The main benefits of multihoming support for the NEWSKY network are

\footnotetext{
${ }^{2}$ The figure is based on ${ }^{7}$. Each network cloud designates one operational domain. The location of Home Agent is just an assumption in order to show the difference between node-based and network-based mobility approaches.
}

for providing seamless handover and load balancing for different types of services.

The third and last module related to mobility is the handover module that is responsible for the following functions:

- Retrieving available radio technologies and their characteristics

- Collecting information from RRM such as link quality, allocation context

- Send appropriate signaling to the routing function so that handover is implemented

- Send appropriate signaling to the mobility function so that handover is anticipated (e.g. fast handover mobility)

As already mentioned before, due to the access technology heterogeneity within NEWSKY, it is important to expose certain characteristics of the link layer to the higher layers. This brings several benefits:

- Proactive handover decisions if it is known that a link is currently degrading and the association to a base station/gateway is about to be lost

- Better handover decision making and network selection if more information on the characteristics of the link technology or on the access network is available

- Ensure that the QoS in the target system can fulfill the required QoS level

- Allow higher layer entities to control certain aspects of the access technologies.

This requires cross-layer interactions and such a framework is currently being defined in the IEEE 802.21 working group that is working on a standard for "Media Independent Handover Services". Although the main focus of the standard is to facilitate handovers between 3GPP and 802 technologies the relevance to the aeronautical context will be demonstrated within the NEWSKY project.

The core of IEEE 802.21 is the so called Media Independent Handover Function (MIHF) that is a functional block between the link and the network layer as shown in Figure 5. 


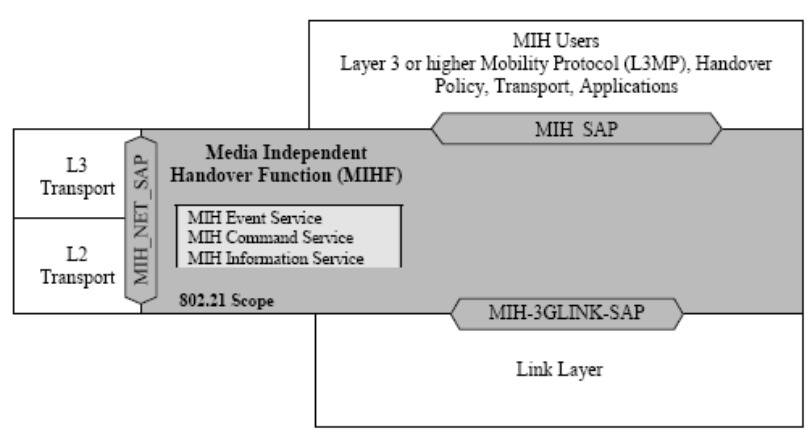

Figure 5. MIHF in the protocol stack. From ${ }^{16}$

Link technologies must implement the 802.21 specific Service Access Points that abstract technology specifics and provide lower layer information in generic form through this standardized interface. Higher layer entities, also called MIH Users, (e.g. the mobility protocol or a handover decision making point) can subscribe to events that signal a state change in the link layer, e.g. a lost association to a base station.

At the same time the MIH Users can send commands to the lower layers that allow to power down a link, to power it up and establish L2 connectivity, etc.

In addition the standard even allows to discover and obtain network information related to availability, static information related to QoS or security among others.

\section{End-to-End Data Transport Functionality}

The end-to-end data transport is working at transport level to ensure end-to-end data delivery, with (e.g. TCP) or without (e.g. UDP) reliability, as well as congestion control at transport level. TCP is assumed to be the transport layer protocol connected to the NEWSKY IP network for most applications. Due to the heterogeneous technologies possibly connected to the NEWSKY network, some adaptations of TCP are necessary depending on the technology used, as it is widely known that TCP performance is highly impacted by wireless technologies due to higher delays, error rates or profiles.

Several alternative architectures are envisaged to support TCP with the different wireless technologies foreseen by NEWSKY ${ }^{1}$ :
- Access technology independent architecture.

This solution uses a standard TCP protocol and not adaptations are done for the different data links and access technologies. The main drawback of this solution is that a strong performance degradation will occur in particular for the satellite links that are the most constraining wireless links.

\section{- Performance Enhancement Proxies (PEP).}

With PEPs the TCP connection is not supported end-to-end but split at airborne and ground routers. TCP protocol is thus optimized for each part of the network and each mobile link technology used then ensuring better end-to-end performance in a multitechnology context, while being transparent to end users. However, PEP introduces additional implementation constraints in particular in the router. Moreover, depending on their location and their level of trustability, PEP could have impacts on other functionalities, such as security and mobility.

\section{- Protocol selection in the ESs.}

The End System directly selects the best TCP version to be used depending on the technology that is used on the mobile link. This solution has several of drawbacks, the main one being the terminal has to implement several protocol stacks and some standard equipments (such as personal device for Aeronautical Passenger Communications) can not be modified and thus could use the respective links only in a very inefficient way.

The two most promising solutions are the two first ones, each with their drawbacks and advantages. Best solution to be retained is further discussed in ${ }^{17}$ and it seems that a combination of the two solutions would be the best trade-off.

Finally, alternative schemes, such as nextgeneration congestion-controlled methods (DCCP), or enhancement (back pressure) or evolution (QuickStart, XCP, ...) of PEP mechanisms for TCP applications are considered. 


\section{Security Functionality}

Security mechanisms are currently being defined following a thread analysis, a detailed requirements definition and an investigation of security service options and mechanisms. Implications of security mechanisms on traffic management, mobility solutions and transport layer adaptations, and vice versa are closely investigated.

\section{Conclusions}

NEWSKY a concept and initial design of a global, seamless aeronautical communication network with focus on air-ground communications and IPv6 technologies, integrating different data links and services. Traffic management, mobility management, security and end-to-end data transport have been identified as the key functionalities. The main results described in this paper are summarized as follows:

- Traffic management: If separated airborne routers for ATS/AOC and APC are used, no CAC is required and a simple static trunk DiffServ architecture could already provide the needed mechanisms to ensure message prioritization and transmission which is compliant with the ATS/AOC requirements.

\section{References}

${ }^{1}$ NEWSKY project website: http://www.newsky-fp6.eu/

2 M. Marchese, "QoS over Heterogeneous Networks”, John Wiley \& Sons, 2007.

${ }^{3}$ ETSI, "Satellite Earth Stations and Systems (SES), Broadband Satellite Multimedia, IP over Satellite”, ETSI Technical Report, TR 101 985, V1.1.2, November 2002.

${ }^{4}$ R. Braden, L. Zhang, S. Berson, S. Herzog, S. Jamin, ,Resource ReSerVation Protocol (RSVP) Version 1 Functional Specification“, IETF RFC 2205, September 1997.
- Mobility management: Proxy Mobile IPv6 (PMIPv6) for local mobility and NEMO for global network mobility are promising solutions for aeronautics, with issues like NEMO Route Optimization to be solved. Furthermore, IEEE 802.21 is an interesting standard for handover optimization in aeronautics.

- End-to-end data transport: The use of Performance Enhancement Proxies (PEP) and the resulting constraints will be necessary for connection oriented satellite communications

\section{Email Addresses}

frank.schreckenbach@dlr.de

katia.leconte@thalesaleniaspace.com

cedric.baudoin@thalesaleniaspace.com

christian.kissling@dlr.de

christian.bauer@dlr.de

serkan.ayaz@dlr.de

\section{ICNS Conference}

5-7 May 2008

${ }^{5}$ J. Wroclawsky, "The Use of the Resource Reservation Protocol with the Integrated Service”, IETF RFC 2210, September 1997.

${ }^{6}$ S. Blake, D. Black, M. Carlson, E. Davies, Z.

Wang, W. Weiss, “An Architecture for

Differentiated Services”, IETF RFC 2475, December 1998.

${ }^{7}$ J.Kempf, "Problem Statement for Network-Based Localized Mobility Management (NETLMM)", RFC 4830, April 2007.

${ }^{8}$ D. Johnson, C. Perkins, and J. Arkko, "Mobility Support in IPv6”, RFC 3775, June 2004.

${ }^{9}$ ICAO ACP WG I-06/WP-08, Updated Mobility Management Requirements for the "Manual for the ATN using IPS Standards and Protocols", March 2008 
10 S. Gundavelli, V. Devarapalli, K. Chowdhury, and B. Patil, "Proxy Mobile IPv6", draft-ietfnetlmm-proxymip6-11 (work in progress), , February 2007.

${ }^{11}$ V. Devarapalli, R. Wakikawa, A. Petrescu, and P. Thubert, "Network Mobility (NEMO) Basic Support Protocol”, RFC 3963, January 2005.

12 R. Wakikawa (Ed.), T. Ernst, K. Nagami, V. Devarapalli (Ed.), "Multiple Care-of Addresses Registration”, draft-ietf-monami6-multiplecoa-06 (work in progress), February 2008.

13 C. Ng, F. Zhao, M. Watari, and P. Thubert, "Network Mobility Route Optimization Solution Space Analysis”, RFC4889, July 2007.

${ }^{14}$ W. Eddy, W. Ivancic, and T. Davis, "NEMO Route Optimization Requirements for Operational Use in Aeronautics and Space Exploration Mobile Networks", draft-ietf-mext-aero-reqs-01 (work in progress), February 2008.

${ }^{15}$ H. Soliman, N. Montavont, N. Fikouras, and K. Kuladinithi, "Flow Bindings in Mobile IPv6 and Nemo Basic Support", draft-soliman-monami6flow-binding-05 (work in progress), November 2007.

${ }^{16}$ IEEE P802.21/D8.0, “Draft Standard for Local and Metropolitan Area Networks: Media Independent Handover Services,” December 2007.

${ }^{17}$ Protocol stack options in heterogeneous aeronautical networks, C. Kissling, C. Baudoin, C. Bauer, ASMS 2008 conference, Bologna, 26-28

August 2008 